\title{
The supernova rate per unit mass
}

\author{
F. Mannucci ${ }^{1}$, M. Della Valle ${ }^{2,3}$, N. Panagia ${ }^{3, \star}$, E. Cappellaro ${ }^{4}$, \\ G. Cresci ${ }^{5}$, R. Maiolino ${ }^{2}$, A. Petrosian ${ }^{6}$, and M. Turatto ${ }^{7}$ \\ ${ }^{1}$ CNR - IRA, Largo E. Fermi 5, 50125 Firenze, Italy \\ e-mail: filippo@arcetri.astro.it \\ 2 INAF, Osservatorio Astrofisico di Arcetri, Largo E. Fermi 5, 50125 Firenze, Italy \\ 3 Space Telescope Science Institute, 3700 San Martin Drive, Baltimore, MD 21218, USA \\ 4 INAF, Osservatorio Astronomico di Capodimonte, salita Moiariello a Capodimonte 16, 80131 Napoli, Italy \\ 5 Dipartimento di Astronomia, Universitá di Firenze, Largo E. Fermi 5, 50125, Firenze, Italy \\ ${ }^{6}$ Byurakan Astrophysical Observatory and Isaac Newton Institute of Chile, Armenian Branch, Byurakan 378433, Armenia \\ 7 INAF, Osservatorio Astronomico di Padova, vicolo dell'Osservatorio 5, 35122 Padova, Italy
}

Received 4 June 2004 / Accepted 17 November 2004

\begin{abstract}
We compute the rate of supernovae ( $\mathrm{SNe}$ ) of different types along the Hubble sequence normalized to the nearinfrared luminosity and to the stellar mass of the parent galaxies. This is made possible by the new complete catalog of nearinfrared galaxy magnitudes obtained by 2MASS. We find that the rates of all SN types, including Ia, Ib/c and II, show a sharp dependence on both the morphology and the $(B-K)$ colors of the parent galaxies and, therefore, on the star formation activity. In particular we find, with a high statistical significance, that the type Ia rate in late type galaxies is a factor $\sim 20$ higher than in E/S0. Similarly, the type Ia rate in the galaxies bluer than $B-K=2.6$ is about a factor of 30 larger than in galaxies with $B-K>4.1$. These findings can be explained by assuming that a significant fraction of Ia events in late spirals/irregulars originates in a relatively young stellar component.
\end{abstract}

Key words. stars: supernovae: general - infrared: galaxies

\section{Introduction}

The supernova (SN) rate normalized to the stellar mass of the parent galaxies contains unique information on the initial mass function of stars in the range of masses between about 3 and $100 M_{\odot}$ (e.g. Madau et al. 1998), therefore it is a very powerful tool for understanding the formation and the chemical evolution of the galaxies and constraining their star formation histories. In particular, the rate of the so called "core-collapse" (CC) supernovae, i.e. type II and Ib/c, which have massive progenitors (e.g. Woosley et al. 2002), reflects the instantaneous birth rate of stars more massive than $8 M_{\odot}$ (e.g. Iben \& Renzini 1983), whereas the trend of the frequency of type Ia SNe from ellipticals to late spirals can provide deep insights in the controversial nature of the binary companion in type Ia events (see for example Hamuy et al. 2003; Livio \& Riess 2003). In this paper we focus our attention on the dependence of the $\mathrm{SN}$ rate on the morphological Hubble type and on the $B-K$ color of the parent galaxies. The empirical grounds for this kind of study are provided by the supernova surveys carried out in the local Universe in the past years (e.g. Cappellaro et al. 1999, hereafter C99)

^ On assignment from the Space Telescope Operations Division, Research and Scientific Support Department of ESA. or still ongoing, such as LOTOSS (Filippenko et al. 2001). Systematic surveys make it possible to compute the SN rates by applying the control time technique (Zwicky 1942) after taking into account the various selection effects (e.g. Cappellaro et al. 1997, hereafter C97). In all cases, the SN rate is normalized to some quantity somehow related to the galaxy "sizes". The quantity most commonly used is the luminosity in the optical $B$ band (e.g. van den Bergh \& Tammann 1991; Tammann et al. 1994), assumed to be a measure of the stellar mass at least for galaxies of the same morphological class (Tammann 1974). Thus, the classical SN unit (SNuB) is defined as number of events per century per $10^{10} L_{\odot}$ in the $B$ band. More recently, C99 and Mannucci et al. (2003) used the far-infrared luminosity, commonly considered to be proportional to the Star Formation Rate (SFR) (e.g., Hirashita et al. 2003), to normalize the $\mathrm{SN}$ rate, thus expressing the rates in SNuIR.

In their most recent determination of the local rates C99 found: a) the rates of types $\mathrm{Ib} / \mathrm{c}$ and II SNe are null in the $\mathrm{E} / \mathrm{S} 0$ galaxies and show a moderate increase from $\mathrm{Sa}$ to $\mathrm{Sd}$ types; $b$ ) a marginally significant decrease of type II rates is also observed from Sd to Irr. $c$ ) the rate of type Ia SNe measured in $\mathrm{SNuB}$ is almost constant along the Hubble sequence from elliptical to Sd galaxies and shows a modest increase 
toward the irregular and peculiar galaxies (admittedly with a low statistical significance). The interpretation of the latter result is not obvious: if type Ia SNe are related to old stellar populations, one would expect a decreasing rate through the whole morphological Hubble sequence from E/S0 to Irr. The problem may be due to the normalization to the $B$ luminosity, which is a poor tracer of the stellar mass along the whole Hubble sequence (see Sect. 2).

In this paper we intend to go beyond this approach and derive the rates of the various types of $\mathrm{SNe}$ normalized to the stellar mass of the parent galaxies as inferred from the $K$-band luminosity. This is now possible thanks to the Two Micron All Sky Survey (2MASS ${ }^{1}$, Jarrett et al. 2003) whose catalog of near-infrared magnitudes of extended objects is now complete. In Sect. 3 the input catalog will be presented, in Sects. 4 and 5 the near-infrared magnitudes and the recipe to compute the mass will be discussed. In the next sections we will present the results and their interpretation.

\section{Importance of the $\mathrm{SN}$ rate per unit mass}

In the past decades the luminosity in the $B$ band has been used as a rough gauge of the mass of the galaxies. Indeed $B$ luminosity has been for long time the only available photometric measurement for most systems observed in the local Universe. Since the presence of a young stellar component can contribute significantly to the $B$ luminosity in the late Hubble type galaxies, the proportionality between $B$ luminosity and stellar mass is expected to change dramatically along the Hubble sequence. As an example, we note that, after using the galaxy colors by Fioc \& Rocca-Volmerange (1999) to compute the M/L ratio as in Bell \& de Jong (2001, hereafter BJ01), a difference of a factor of 10 is found in the stellar mass between an elliptical and an irregular galaxy with the same $B$ magnitude and colors typical of their classes. Large differences can also be found within each class of galaxies: as an example, a range of a factor of 3 in mass is found for Sb galaxies having $(B-K)$ colors within $\pm 1 \sigma$ of the average of their class.

In addition we note that the $B$ flux is the result of combined emission from old stars, emission from young populations, and absorption by dust, with the relative contributions changing along the Hubble sequence. Even if $B$ light would be an acceptable measure of the stellar mass in the E/S0 galaxies, it is a very poor tracer of mass along the whole Hubble sequence.

In the past years, a number of authors (van den Bergh 1990; Della Valle \& Livio 1994; Panagia 2000) had normalized the rate of type Ia SNe to the near-infrared $H$ and $K$ bands, which are better tracers of stellar mass than $B$ light. They found a sharp increase of the production of type Ia SNe toward late morphological types (see also Mannucci et al. 2003). However, in those the computation of the normalized SN rate was carried

\footnotetext{
1 The Two Micron All Sky Survey is a joint project of the University of Massachusetts and the Infrared Processing and Analysis Center/California Institute of Technology, funded by the National Aeronautics and Space Administration and the National Science Foundation.
}

out by using average colors for the parent galaxies, because at that time the individual near-infrared measurements were not available for most galaxies. Recently, the 2MASS collaboration released their catalog (Jarrett et al. 2003), and now an accurate normalization of the $\mathrm{SN}$ rate to the total stellar mass has become possible.

\section{3. $\mathrm{SN}$ catalog}

In this study we used the $\mathrm{SN}$ catalog from C99. This sample is a compilation of $136 \mathrm{SNe}^{2}$ discovered by five groups: the SN search of the Padova group with the Asiago Schmidt telescopes, the Sternberg Institute search at the Crimea observatory, the visual search by R. Evans, the survey by the Observatoire of the Côte d'Azur, and the Calan/Tololo search. The full descriptions of these searches and the relative references are given in C97. These searches were chosen because all the information for the computation of the control time and of the observational bias, such as the galaxy sample, the frequency of the observations and the limit magnitudes, were available. Only SNe in galaxies of known luminosity, colors, morphology, inclination and distance can be considered in our study. Therefore, even if the merging of the five original catalogs yields about $250 \mathrm{SNe}$, the sample used by C99 includes only 136 events that have occurred in galaxies reported in the RC3 catalog (de Vaucouleurs et al. 1991) and in the LEDA database $^{3}$. The $\mathrm{SNe}$ in the final catalog have an average distance of $38 \mathrm{Mpc}$, with $90 \%$ of them below $85 \mathrm{Mpc}$.

The computational procedure to correct for the selection effects is described in C97 and is based on several ingredients such as the absolute magnitude at maximum, the light evolution in the $B$ band, the average extinction, the correction for inclination of the spiral galaxy with respect to the line of sight, the fraction of SNe lost in the overwhelming brightness of the galaxy nuclei. For consistency, we used the same assumptions and procedures as in C97 and C99.

\section{The near-infrared magnitudes}

We cross-correlated the catalog of the 9346 galaxies of C99 with the 2MASS Extended Source Catalog (XSC) at IRSA ${ }^{4}$ to obtain the near-IR magnitudes. The coordinates were obtained from the HyperLeda catalog ${ }^{5}$ (Paturel et al. 1989; Prugniel \& Héraudeau 1998). Among the possible choices of photometric measurements in the 2MASS catalog, we used the total photometry obtained by extrapolating the fit of a Sersic function to the galaxy radial profile $\left(k \_m \_\right.$ext in the 2MASS nomenclature, see Jarrett et al. 2003, for details). This is the photometric measurement best suited to represent the total magnitude to be compared with the RC3 total $B$ magnitudes. We retrieved 8349 galaxies present in the 2MASS XSC, i.e., $89 \%$

\footnotetext{
2 One of the 137 SNe used in the C99 was later discovered to be associated to a galaxy not included in the sample.

The Lyon-Meudon Extragalactic Database (LEDA) is supplied by the LEDA team at the CRAL-Observatoire de Lyon (France).

4 http://irsa.ipac. caltech.edu

5 http://leda.univ-lyon 1. fr
} 


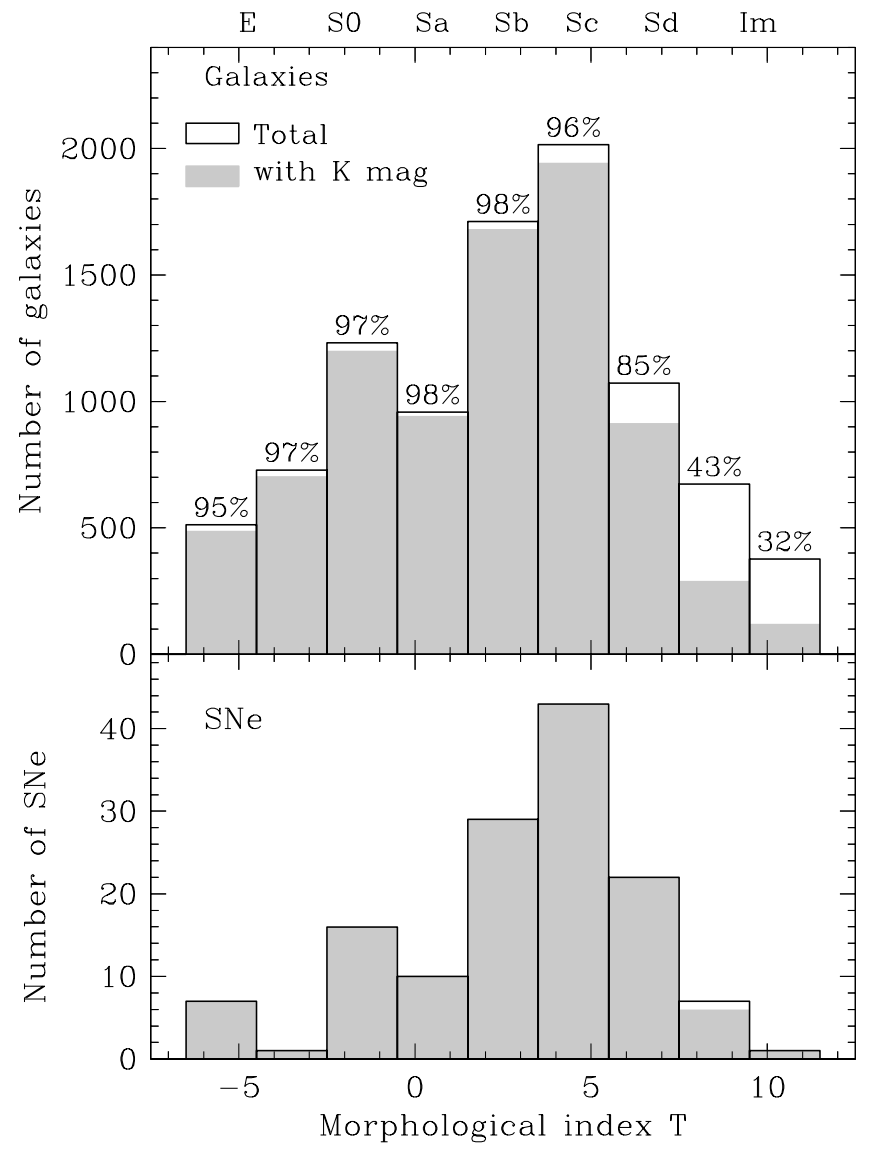

Fig. 1. Upper panel: the histogram of the number of galaxies in the input $\mathrm{C} 99$ catalog (in white) as a function of the morphological index $T$ is compared with that of the galaxies having a 2MASS detection in $K$ (in gray). The fraction of detected galaxies is shown over each bin. Lower panel: histogram of the number of $\mathrm{SNe}$ as a function of the morphological index of the parent galaxy. In white the input C99 catalog, in grey the catalog with retrieved $K$-band magnitude.

of the input catalog. Figure 1 shows the distribution of the morphological indices of the galaxies with available $\mathrm{K}$ magnitudes compared to the distribution of the original C99 catalog (upper panel). It shows that the 2 MASS catalog contains the nearinfrared magnitudes of essentially all the galaxies with morphological types between $\mathrm{E}$ and $\mathrm{Sd}$, while about $2 / 3$ of the Irr are missing. Most of them are actually present at a faint level in the 2MASS images, at least in J, but have not been retrieved by the XSC software.

We can estimate an upper limit to the contribution to the total $K$-band luminosity from the galaxies not detected by 2 MASS assuming they all have a $K$-band magnitude equal to the survey limit for extended objects of $K=13.5$ (see www.ipac.caltech.edu/2mass/overview/ about2mass.html). Figure 2 shows the resulting absolute magnitudes: most of the galaxies undetected by 2MASS are local dwarf galaxies, small systems which are bright in the $B$ band but faint in the $K$ band. Their contribution to the total $K$-band luminosity of the total galaxy sample is below the tiny fraction of $0.3 \%$, and it is below $5 \%$ even if only the Irr galaxies are considered. For this reason the incompleteness of

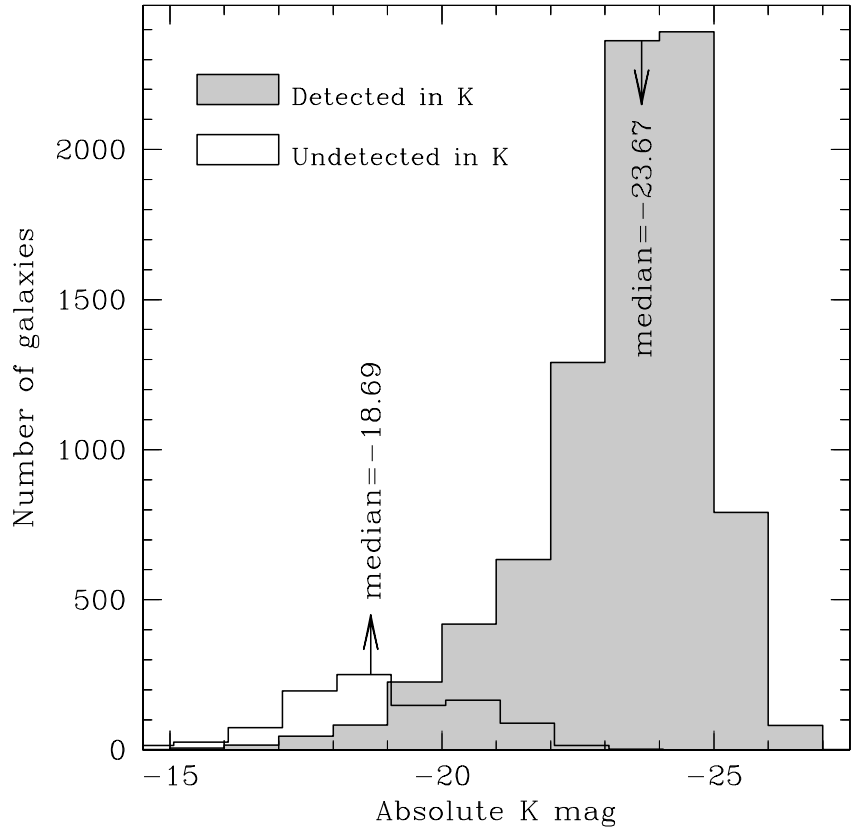

Fig. 2. The absolute $K$-band magnitude of the galaxies detected by 2MASS is compared to that of the undetected galaxies (see text). It is apparent that most the of galaxies missing from the 2MASS catalog are local dwarfs contributing very little to the total $K$-band luminosity and mass. The difference between the median of the two distribution is about $5 \mathrm{mag}$.

Table 1. Number of SNe and galaxies per morphological bin.

\begin{tabular}{lrrrr}
\hline \hline Type & Ngal & Ia & Ib/c & II \\
\hline E/S0 & 2048 & 21.0 & 0 & 0 \\
S0a/b & 2911 & 18.5 & 5.5 & 16.0 \\
Sbc/d & 2682 & 21.4 & 7.1 & 31.5 \\
Irr & 644 & 6.8 & 2.2 & 5.0 \\
\hline
\end{tabular}

the 2MASS XSC catalog is not a serious problem for the computation of the rates.

This is confirmed by the fact that only $1 \mathrm{SN}$ out of the input list of 136 events occurred in a galaxy not present in the 2MASS catalog. This SN was therefore removed from the sample (see Fig. 1, lower panel).

For 64 galaxies $(0.77 \%$ of the sample) the morphological classification is absent or very uncertain. These galaxies were excluded from the sample when the $\mathrm{SN}$ rate was computed as a function of the morphology (Sect. 6) but were used to compute the $\mathrm{SN}$ rate as a function of the $B-K$ color (Sect. 7).

The final numbers of $\mathrm{SNe}$ and galaxies used in our computations are reported in Table 1. The non integer numbers of SNe are due to the presence of a few $\mathrm{SNe}$ with incomplete or unknown classification which were divided into different classes, as explained in $\mathrm{C} 97$.

\section{Galaxy mass}

The total stellar masses of the galaxies can be derived from the observed broad-band fluxes by fitting them with spectrophotometric galaxy evolution models (e.g., Brinchmann \& Ellis 2000; Dickinson et al. 2003). When the redshift and the 
morphological type of a galaxy are known, these methods are expected to give rather accurate results: for a given Initial Mass Function (IMF) the typical uncertainties in the total masses are less than $40 \%$. Larger uncertainties, up to a factor of two, are associated to the IMF as the low mass stars give a dominant contribution to the total mass and a secondary one to the luminosity. For example a difference of a factor of two in galaxy mass is found between the Salpeter (1955) IMF, which is rich in low-mass stars, and the Kroupa (2001) IMF, which has a shallower slope for masses below $0.5 M_{\odot}$. Fortunately, as long as no systematic differences of IMF are present along the Hubble sequence, this uncertainty only has the effect of shifting up and down the total mass without introducing relative changes along the Hubble sequence.

We estimate the mass of the galaxies by using the method developed by BJ01. These authors computed the mass-to-light ratio $(\mathrm{M} / \mathrm{L})$ by using galaxy evolutionary synthesis models and found a tight correlation between $\mathrm{M} / \mathrm{L}$ and the optical-to-nearIR colors: for a given $K$-band magnitude, bluer galaxies are less massive because they have younger populations. Therefore, the stellar mass can be derived from the $K$-band luminosity and the $B-K$ color, which is an indicator of the mean age of the population. Based on the use of the luminosities in two bands only, this method can be applied to large samples of galaxies with data available for a limited number of filters. This is our case as most of the galaxies monitored during the $\mathrm{SN}$ searches have $B$ and $K$-band magnitudes available.

As mentioned above, the stellar mass derived depends on the adopted IMF which cannot be easily constrained from the data and therefore must be assumed a priori. BJ01 use a Salpeter IMF and scale down by a factor of 0.7 the resulting $\mathrm{M} / \mathrm{L}$ ratio to obtain "maximum disk" mass, i.e., the maximum mass compatible with the observed rotation curve.

BJ01 constructed their models to reproduce the properties of the spiral galaxies. These galaxies show a wide range of properties, from the quiescent $\mathrm{S} 0$ to the very active $\mathrm{Sd}$, and this is why these models can produce a wide range of colors. For example, the $B-V$ color range covered by the BJ01 models goes from 0.29 to 0.95 , corresponding, respectively, to the average color of the irregulars $(B-V=0.27)$ and of the ellipticals $(B-V=0.96$, Fukugita et al. 1995). As a consequence these models can be used to compute the $\mathrm{M} / \mathrm{L}$ ratio along the whole Hubble sequence, from ellipticals to irregulars.

BJ01 tested the robustness of their method by using several independent evolutionary models, and studied the effect of introducing secondary bursts of star formation (galaxies become brighter but bluer) or dust extinction (galaxies become fainter but redder). Apart from the effect of the IMF, the uncertainties in the $\mathrm{M} / \mathrm{L}$ from this method are about 0.4 dex peak-to-peak.

From the coefficients provided in Table 1 of BJ01 we derived the relation giving the stellar mass from the $K$-band luminosity $L_{K}$ and the total color $(B-K)$ :

$\log \left(\frac{M / L_{K}}{M_{\odot} / L_{\odot}}\right)=0.212(B-K)-0.959$.

This equation was used to compute the stellar mass of each galaxy. The resulting distributions are shown in Fig. 3 where the galaxies are divided into four morphological classes.

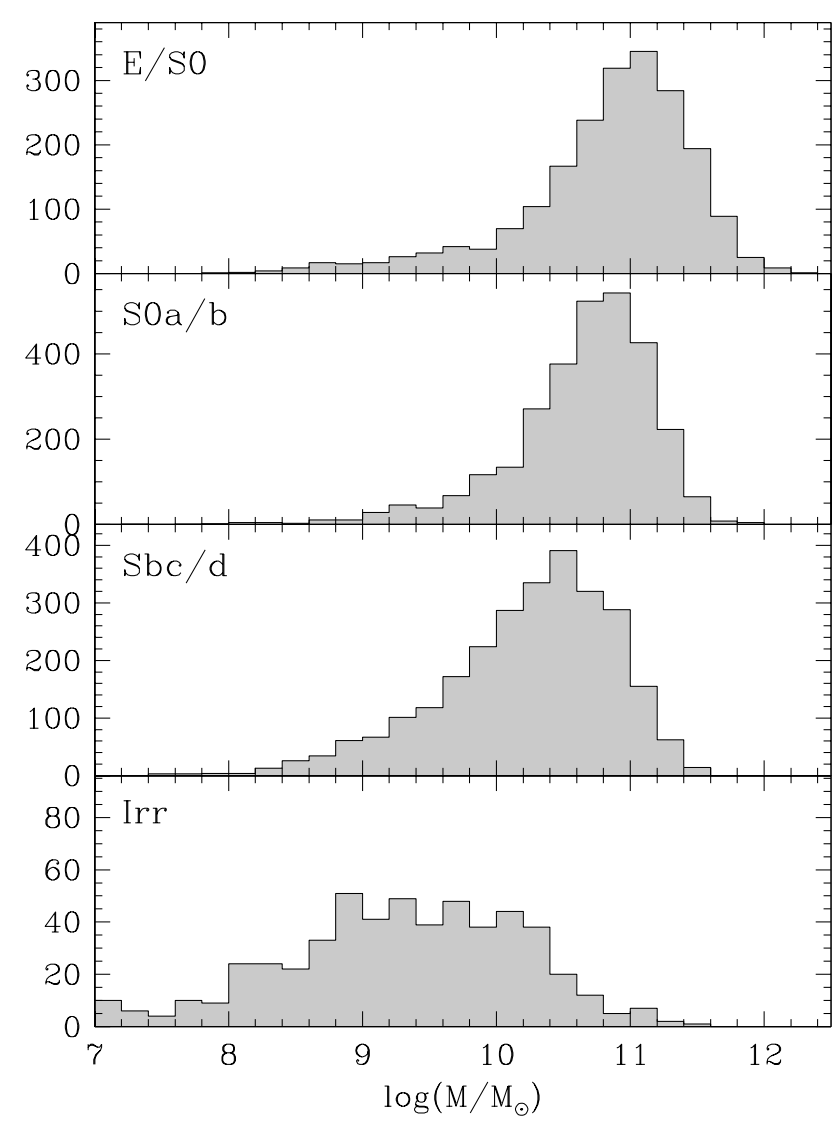

Fig. 3. Distribution of galaxy stellar mass for the different morphological classes.

As a consistency check, we compared the mass distribution of our galaxy sample with that derived by Kauffmann et al. (2003) for 120000 local galaxies observed by the Sloan Digital Sky Survey (SDSS). These authors use a completely different method based on two stellar absorption indices, the $4000 \AA$ break and the Balmer line $\mathrm{H} \delta$. Despite the differences in the selection of the galaxy samples and in the methods to estimate the masses, the two distributions are remarkably similar: in the SDSS sample, half of the total stellar mass is contained in galaxies of less than $3.1 \times 10^{10} M_{\odot}$, which is in fair agreement with the $3.7 \times 10^{10} M_{\odot}$ for the present sample. In both cases the peak of the contribution to the total stellar mass comes from galaxies of about $6 \times 10^{10} M_{\odot}$. This also implies that the present sample is a fair representation of the local universe as observed in the optical by modern wide-area surveys as the SDSS. The results are also consistent with those derived from models based on a larger number of photometric bands (e.g., Saracco et al. 2004; Drory et al. 2004).

\section{The $\mathrm{SN}$ rate as a function of the morphology of the host galaxy}

The SN rates computed both in units of $K$-band luminosity and stellar mass are shown in Table 2 and in Fig. 4. In the first case the rates are expressed in number of SNe per century per $10^{10}$ $K$-band solar luminosity ( $\mathrm{SNuK}$ ), using a $K$ band magnitude of the Sun of 3.41 (Allen 1973) corresponding to a solar luminosity in the $K$ band of $L_{K, \odot}=5.67 \times 10^{31} \mathrm{erg} / \mathrm{s}$. In the second case 
Table 2. SN rates normalized to the $K$-band luminosity and to the stellar mass. The errors are $1 \sigma$ values and contain the contribution of the Poisson statistics of the SN number, often dominant, and of several other uncertainties, as explained in C97. The upper limits correspond to $90 \%$ confidence levels.

\begin{tabular}{lccc}
\hline \hline Type & Ia & Ib/c & II \\
\hline \multicolumn{4}{c}{ SN rate per $K$-band luminosity $(\mathrm{SNuK})$} \\
\hline $\mathrm{E} / \mathrm{S} 0$ & $0.035_{-0.011}^{+0.013}$ & $<0.0073$ & $<0.10$ \\
$\mathrm{~S} 0 \mathrm{a} / \mathrm{b}$ & $0.046_{-0.017}^{+0.019}$ & $0.026_{-0.013}^{+0.019}$ & $0.088_{-0.039}^{+0.043}$ \\
$\mathrm{Sbc} / \mathrm{d}$ & $0.088_{-0.032}^{+0.035}$ & $0.067_{-0.032}^{+0.041}$ & $0.40_{-0.16}^{+0.17}$ \\
$\mathrm{Irr}$ & $0.33_{-0.13}^{+0.18}$ & $0.21_{-0.14}^{+0.26}$ & $0.70_{-0.43}^{+0.57}$ \\
\hline \multicolumn{4}{c}{$\mathrm{SN} \mathrm{rate} \mathrm{per} \mathrm{Mass}(\mathrm{SNuM})$} \\
\hline E/S0 & $0.044_{-0.014}^{+0.016}$ & $<0.0093$ & $<0.013$ \\
$\mathrm{~S} 0 \mathrm{a} / \mathrm{b}$ & $0.065_{-0.025}^{+0.027}$ & $0.036_{-0.018}^{+0.026}$ & $0.12_{-0.054}^{+0.059}$ \\
$\mathrm{Sbc} / \mathrm{d}$ & $0.17_{-0.063}^{+0.068}$ & $0.12_{-0.059}^{+0.074}$ & $0.74_{-0.30}^{+0.31}$ \\
Irr & $0.77_{-0.31}^{+0.42}$ & $0.54_{-0.38}^{+0.66}$ & $1.7_{-1.0}^{+1.4}$ \\
\hline
\end{tabular}

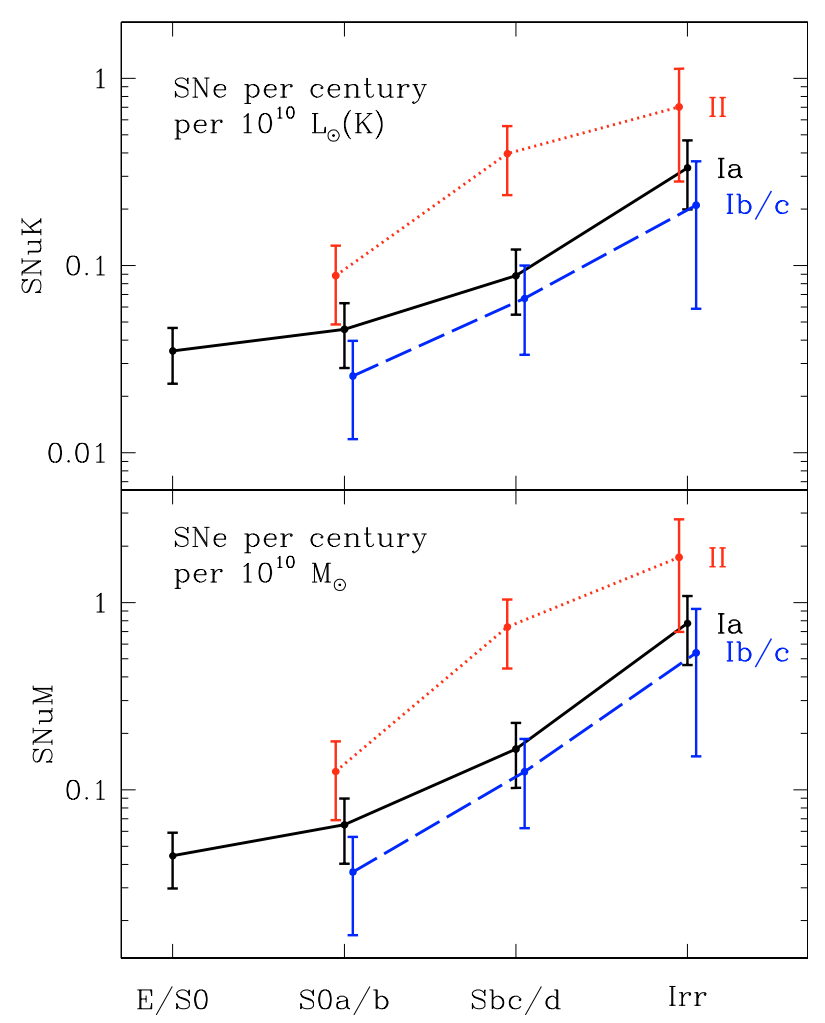

Fig. 4. Upper panel: $\mathrm{SN}$ rate per $K$ band luminosity as a function of morphological index expressed in SNuK (number of SN per century per $10^{10} L_{\odot}$ of luminosity in the $K$ band). The lines correspond to type Ia (solid), type II (dotted), and Ib/c (dashed) with $1 \sigma$ error bars. Lower panel: SN rate normalized to the stellar mass and expressed in $\mathrm{SNuM}$, i.e., number of SNe per century per $10^{10} M_{\odot}$.

they are expressed in number of SNe per century and per $10^{10}$ solar masses in stars (SNuM). The errors include the Poisson errors due to the $\mathrm{SN}$ statistics as well as the uncertainties on the input parameters and on the bias corrections as explained in C97 and C99. For the first time these rates are derived in a fully consistent way by using the $\mathrm{M} / \mathrm{L}$ ratios derived from
Table 3. Number of SNe, number of galaxies and $\mathrm{SN}$ rate per unit mass in SNuM per color bin. The upper limit is at $90 \%$ confidence level.

\begin{tabular}{crrrcc}
\hline \hline$B-K$ & Ngal & Ia & CC & SNr(Ia) & SNr(CC) \\
\hline$<2.6$ & 1499 & 9.0 & 20.0 & $0.86_{-0.35}^{+0.45}$ & $2.3_{-0.77}^{+0.86}$ \\
$2.6-3.3$ & 2178 & 15.4 & 29.6 & $0.19_{-0.07}^{+0.08}$ & $0.41_{-0.12}^{+0.13}$ \\
$3.3-4.1$ & 3396 & 37.3 & 17.7 & $0.084_{-0.025}^{+0.026}$ & $0.043_{-0.015}^{+0.017}$ \\
$>4.1$ & 1276 & 6.0 & 0 & $0.027_{-0.013}^{+0.017}$ & $<0.012$ \\
\hline
\end{tabular}

the color of each galaxy and not average M/L ratios (Tammann 1974) or average colors (Panagia 2000; Mannucci et al. 2003).

The rates for the Irr galaxies have large uncertainties because of the small sample of SNe. Nevertheless, a clear increase of the $\mathrm{SN}$ rates from $\mathrm{E} / \mathrm{S} 0$ to $\mathrm{S} 0 \mathrm{a} / \mathrm{b}$ to $\mathrm{Sbc} / \mathrm{d}$ to Irr can be seen for all the SN types. In particular, the lower panel of Fig. 4 shows that all types of SNe in spirals and Irr have a remarkably similar behavior, with the rates in the Irr galaxies being 12-15 times larger than those in the SOa/b galaxies (see Table 2).

Qualitatively the result is the same as that derived by Mannucci et al. (2003) by using average colors, but the difference of rates along the Hubble sequence is even more pronounced: there the SN Ia rate in the Sbc/d galaxies was found to be a factor of 2 higher than that in the E/S0, to be compared with the factor of 4 of the present work, and that in the Irr galaxies a factor of 7 higher than in E/S0, to be compared with the factor of $\sim 17$ found here.

Despite the small number of $\mathrm{SNe}$, the statistical significance of the difference between the type Ia rate in E/S0 and late spirals/irregulars is high: the rate observed in E/S0 would correspond to $0.4 \mathrm{SNe}$ for the Irr galaxies, instead of the observed number of 6.8: such a value is excluded by the Poisson statistics at a significance level higher that 0.9995 When the other sources of errors are also included, the final significance remains above $99 \%$.

\section{The $\mathrm{SN}$ rate as a function of the galaxy colors}

The SFR is related both to the morphology and to the color of the galaxies (e.g., Kennicutt 1998). These are not one-toone relations, as the dust content, the star formation history and the presence of recent mergers introduce large spreads. Nevertheless the SFR-color relation is probably tighter than the SFR-morphology one as the color is more directly related to stellar population than is the morphology. For this reason we computed the SN rates also after binning the galaxies according to their $B-K$ colors. To increase the number of objects in each color bin we add up two types of CC SNe.

The results are shown in Table 3 and plotted in Fig. 5. It is evident that the rate of the CC SNe has a strong dependence on the galaxy colors, as already noted, e.g., by C99 when normalizing to the $B$ band. The rate of Type Ia SNe also changes rapidly with colors, although not as quickly as for the CC SNe. This dependence is produced by the normalization to the stellar mass as it is not present in C99. It also differs from the results 


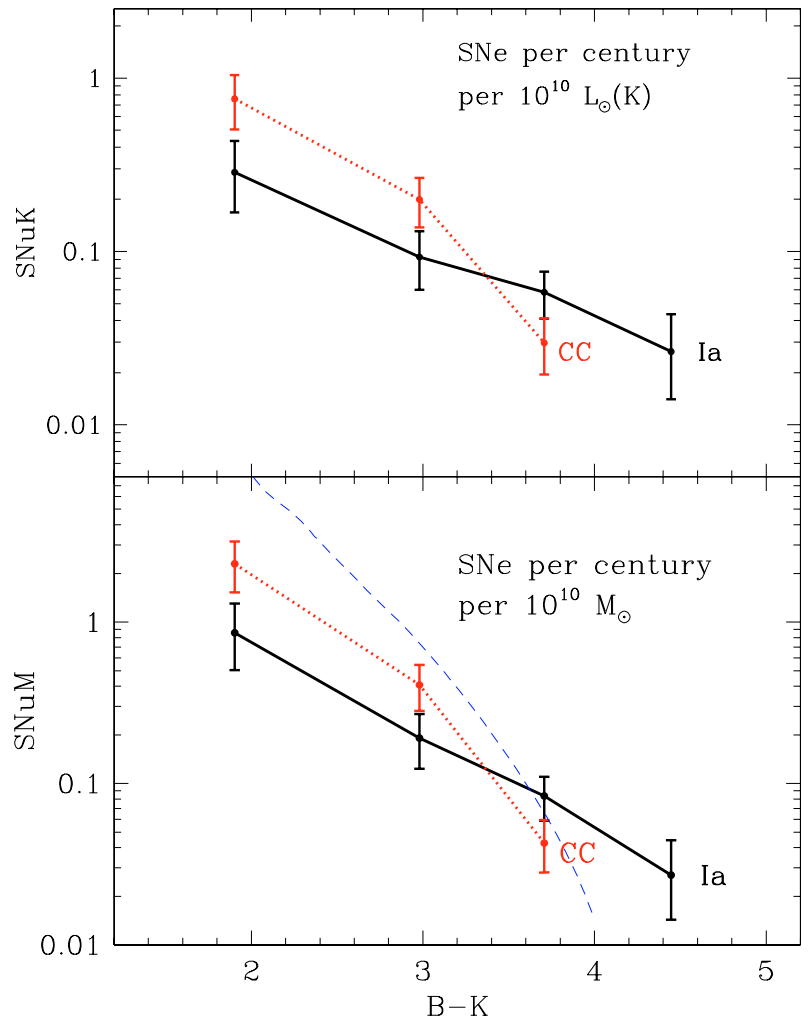

Fig. 5. Upper panel: $\mathrm{SN}$ rate per $K$ band luminosity expressed in $\mathrm{SNuK}$ (number of SN per century per $10^{10} L_{\odot}$ of $K$-band luminosity) as a function of the $B-K$ color of the parent galaxies. The thick lines are the results for type Ia (solid) and core-collapse (dotted). Lower panel: SN rate normalized to the stellar mass and expressed in SNuM, i.e., number of SNe per century per $10^{10} M_{\odot}$ of stellar mass. The thin dashed line shows the relation between the $B-K$ color and the CC SN rate as predicted from a model of spiral galaxy (see text) for ages between 0.7 and $10 \mathrm{Gyr}$ after the onset of an exponentially declining star formation with e-folding time of $2 \mathrm{Gyr}$. These two ages correspond, respectively, to Irr and E galaxies, whose average colors are $B-K=2.23$ for Irr and $B-K=3.98$ for E (Fioc \& Rocca-Volmerange 1999).

by Turatto et al. (1994) who, on the basis of a limited sample of $5 \mathrm{SNe}$, studied the type Ia SN rate in early type galaxies, finding no dependence on any properties of the parent galaxies, such as ISM content in gas or dust. The variation of the $\mathrm{SN}$ rates with the galaxy color is discussed in detail in the next section.

The thin dashed line in the lower panel of Fig. 5 shows the relation between the $B-K$ color and the CC SN rate expected from a simple model of a spiral galaxy. To obtain this, first we computed the expected relation between the $B-K$ color and the SFR by using the GALAXEV models (Bruzual \& Charlot 2003) with solar metallicity and an exponentially declining SFR with an e-folding time of 2 Gyr. This model can reproduce the properties of a wide range of galaxies, from early-type spirals to irregulars, just by selecting the appropriate age since the onset of the star formation (see, for example, Pozzetti et al. 1996). Second, the SFR was converted into an expected SN rate by integrating the IMF for masses between 8 and $40 M_{\odot}$ (see, for example, Madau et al. 1998). As a consequence we have no free parameter in plotting this line. The model considered is very simple, as it includes only one star formation history, only solar metallicity and no dust. Nevertheless it is evident from Fig. 5 that it accurately predicts the behavior of the CC SN rate as a function of galaxy color, confirming that the progenitors of the CC SNe are young populations related to the ongoing star formation. It is also evident that the number of $\mathrm{CC} \mathrm{SNe} \mathrm{ac-}$ tually observed is lower by about a factor of 2 than the model predictions. This is not unexpected as the model considered here is very simple: as an example, reducing the metallicity of the galaxies to $40 \%$ solar would produce galaxies with colors bluer by about $B-K \sim 0.3$, greatly reducing the discrepancy. Nevertheless, this is also consistent with the claims from nearIR SN searches (as Mannucci et al. 2003) that a considerable fraction of the SN expected CC SNe are missing from the optical searches because of the presence of large dust extinction.

\section{Discussion}

We have used the near-infrared photometry by 2MASS to compute the SN rates normalized to both the $K$ luminosity and the mass in stars of the galaxies. We group the parent galaxies either according to their Hubble types (Fig. 4 and Table 2) or to their $B-K$ color (Fig. 5 and Table 3 ). A close inspection of these figures reveals the following:

1 The rate of type Ia, Ib/c and II SNe per unit of mass increases by factors of 2, 3 and 5 respectively from early to late spirals. This is universally accepted for the CC SNe whose rates are known to be closely related to the SFR and not to the total stellar mass. For type Ia SNe this is a more controversial point, as discussed below.

2 The behavior of the mass-normalized rates of various SN classes are remarkably similar, as shown by the slopes of the curves in the bottom panels of Figs. 4 and 5. This finding seems to be at odds with the standard picture for the progenitors of type Ia SNe. The parent binary system could be either single-degenerate or double-degenerate (e.g., Woosley \& Weaver 1986; Wheeler \& Harkness 1993; Branch et al. 1995, 2001; Hoflich et al. 1995; Yungelson \& Livio 2000; Nomoto 2003) and both cases suggest an evolved progenitor. Our result indicates that an "evolved" binary system is not synonymous with a "very old" binary system. On the contrary Figs. 4 and 5 suggest that most progenitors of type Ia $\mathrm{SNe}$ occurring in late spirals and irregulars are associated with a relatively young stellar component (cf. Greggio $\&$ Renzini 1983). We note that this scenario has already been proposed on theoretical grounds by Dallaporta in the early 70's (Dallaporta 1973), while the first evidence for it was found soon after by Oemler \& Tinsley (1979).

Our results can also be expressed in terms of the delay time between a newly born white dwarf (i.e. the primary) and the $\mathrm{SN}$ explosion (indicated by $\tau$ in the parametrization by Madau et al. 1998). Since the time scale of the stellar evolution for stars near $8 M_{\odot}$ is about $50 \mathrm{Myr}$, our results lend support to the models of progenitors characterized by a "short" delay time. This point will be discussed in detail in a forthcoming paper (Mannucci et al. 2004, in preparation). 
3 The rate of type Ia SNe per unit mass increases by a factor of about 4 from E/S0 to Sbc/d and up to a factor of about 17 in Irr galaxies. The same effect can also be seen when the galaxies are binned according to their colors: the ratio between the type Ia SN rate in galaxies bluer than $B-K=2.6$ and redder than $B-K=4.1$ is larger than 30 . Both these results have a very high statistical significance (larger than 0.99 confidence level).

The existence of an order of magnitude difference in the rates between late spirals/irregulars and ellipticals implies that the frequency of binary systems exploding as a type Ia SN per unit time changes appreciably when the parent population ages. This is not totally unexpected in the theoretical framework of the double degenerate scenario. For example Livio (2001) (see also Yungelson et al. 1994) predicts a decrease of a factor of 10 of the merger rate in the "old" $\left(10^{10} \mathrm{yr}\right)$ stellar population with respect to the "young" $\left(10^{8} \mathrm{yr}\right)$ stellar component.

4 However, this might represent only one side of the coin. The aging of the parent population affects not only the SN rate but also the properties of the $\mathrm{SN}$ explosions. Li et al. (2001) have shown that the class of Ia SNe are characterized by a large number of "peculiar" objects $(36 \% \pm 9 \%)$. This high degree of inhomogeneity reflects the existence of systematic differences in the properties of the $\mathrm{SN}$ explosion, such as the velocity of the ejecta (Filippenko 1989; Branch \& van den Bergh 1993; Nugent et al. 1995), the luminosity and photometric evolution (Della Valle \& Panagia 1992; Phillips 1993; Hamuy et al. 1996, 2000; Canal et al. 1996; Howell 2001), and the rate of occurrence (Della Valle \& Livio 1994). Howell (2001) has shown that underluminous SNe-Ia, like 1991bg (Filippenko et al. 1992; Turatto et al. 1996) or SN 1992K (Hamuy et al. 1994) are twice as common in early-type galaxies as in late-type galaxies, whereas overluminous SNe-Ia are more common in late-type galaxies than in early type galaxies (15 vs. 2). Our results are consistent with a picture in which the underluminous, $1991 \mathrm{bg}$ like type Ia SNe are preferentially associated to an old stellar population, whereas overluminous type Ia SNe tend to occur in spiral and star-forming galaxies.

5 The $\mathrm{SN}$ rate as a function of the parent galaxy color in Fig. 5 can be used to estimate the relative contribution of the "old" and "young" channels. The behavior of the SN rate can be reproduced by combining a constant contribution (i.e., independent of galaxy colors) from "old" progenitors and a contribution proportional to the SFR from the "young" progenitors. The first contribution must be similar to the value measured in the reddest (i.e., more quiescent) galaxies or in the ellipticals, while the other contribution must show a behavior similar to that of the $\mathrm{CC} \mathrm{SNe}$. The result of this simple model is shown in Fig. 6: the type Ia rate is well reproduced by the constant rate of $0.044 \mathrm{SNuM}$ measured in the E/S0 plus a fixed fraction (40\%) of the rate of the CC SNe.

The best fitting agreement between the two curves is obtained for:

$\mathrm{SNr}(\mathrm{Ia})=(0.047 \pm 0.009)+(0.35 \pm 0.08) \cdot \mathrm{SNr}(\mathrm{CC})$

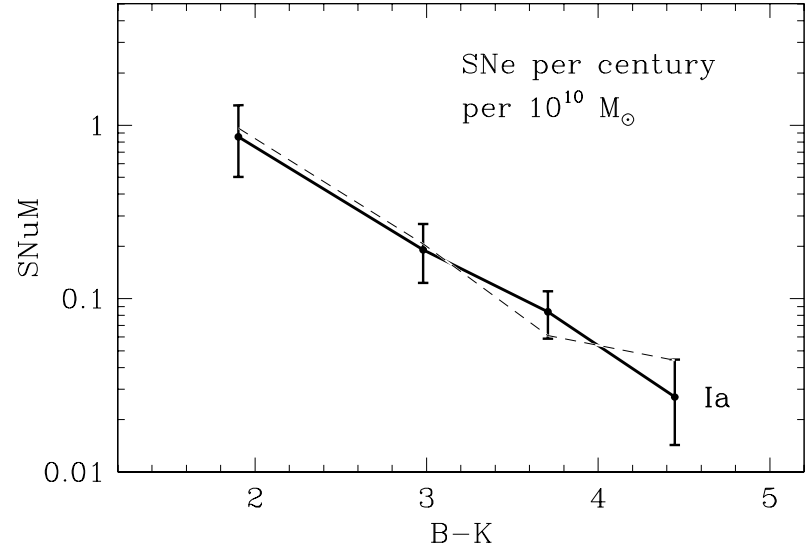

Fig. 6. The rate of the type Ia SNe (thick solid line) is compared with the result of a toy model (thin dashed line) in which the rate is reproduced by a constant value, independent of color and fixed at the value measured in the ellipticals, plus $40 \%$ of the rate of the CC SNe (see Fig. 5). The model reproduces the observed rate well.

where the errors are the formal fitting uncertainties in the two free parameters. These values can also reproduce the type Ia $\mathrm{SN}$ rate as a function of the morphological type in Fig. 4 with great accuracy.

The first term is due to the "old" progenitors, responsible of most SNe-Ia in the ellipticals, about $50 \%$ in S0a/b, about $20 \%$ in Sbc/d and a few \% in the Irr. The second term is roughly proportional to the SFR (assuming that the CC SN rate tracks the instantaneous SFR) and dominates in the late-type galaxies.

We note that the type Ia SN rate in perfectly quiescent galaxies, corresponding to the first (constant) term in Eq. (2), could be even lower. Indeed, the type Ia $\mathrm{SN}$ rate in radio-loud early type galaxies appears to be enhanced, by a factor $\sim 4$, with respect to the radio-quiet sample (Della Valle \& Panagia 2003; Della Valle et al. 2004). The latter $\left(0.023_{-0.008}^{+0.012}\right)$ is similar to the value measured here for the reddest galaxies $\sim 0.027 \mathrm{SNuM}$, (see Table 3). In conclusion, all of this provides (additional) empirical support for the idea, proposed in the past years (Della Valle \& Livio 1994; Ruiz-Lapuente et al. 1995) that SNe Ia in late- and early-type galaxies may originate from different types of progenitors and/or through different explosive channels.

\section{References}

Allen, C. W. 1973, Astrophysical Quantities (London: The Athlone Press)

Bell, E. F., \& de Jong, R. S. 2001, ApJ, 550, 212 (BJ01)

Branch, D. 2001, PASP, 113, 169

Branch, D., \& van den Bergh, S. 1993, AJ, 105, 2231

Branch, D., Livio, M., Yungelson, L. R., Boffi, F. R., \& Baron, E. 1995, PASP, 107, 1019

Brinchmann, J., \& Ellis, R. S. 2000, ApJ, 536, 77

Bruzual, G., \& Charlot, S. 2003, MNRAS, 344, 1000

Canal, R., Ruiz-Lapuente, P., \& Burkert, A. 1996, ApJ, 456, L101

Cappellaro, E., Turatto, M., Tsvetkov, D. Yu., et al. 1997, A\&A, 322, 431 (C97)

Cappellaro, E., Evans, R., \& Turatto, M. 1999, A\&A, 351, 459 (C99)

Dallaporta, N. 1973, A\&A, 29, 393

Della Valle, M., \& Livio, M. 1994, ApJ, 423, L31

Della Valle, M., \& Panagia, N. 1992, AJ, 104, 696 
Della Valle, M., \& Panagia, N. 2003, ApJ, 587, L71

Della Valle, M., Panagia, N., Padovani, P., et al. 2004, ApJ, submitted de Vaucouleurs, G., de Vaucouleurs, A., Corwin, H. G., et al. 1991, Third Reference Catalogue of Bright Galaxies (New-York: Springer-Verlag) (RC3)

Dickinson, M., Papovich, C., Ferguson, H. C., \& Budavári, T. 2003, ApJ, 587, 25

Drory, N., Bender, R., Feulner, G., et al. 2004, ApJ, 608, 742

Filippenko, A. V, Richmond, M., Branch, D., et al. 1992, AJ, 104, 1543

Filippenko, A. V., Li, W. D., Treffers, R. R., et al. 2001, in Small Telescope Astronomy on Global Scales, IAU Coll., 183, ed. B. Paczynski, W.-P. Chen, \& C. Lemme, ASP Conf. Ser., 246, 121

Fioc, M., \& Rocca-Volmerange, B. 1999, A\&A, 351, 869

Greggio, L., \& Renzini, A. 1983, A\&A, 118, 217

Hamuy, M., Phillips, M. M., Maza, J., et al. 1994, AJ, 108, 2226

Hamuy, M., Phillips, M. M., Suntzeff, N. B., et al. 1996, AJ, 112, 2391

Hamuy, M., Trager, S. C., Pinto, P. A., et al. 2000, AJ, 120, 1479

Hamuy, M., Phillips, M. M., Suntzeff, N., et al. 2003, Nature, 424, 651

Hirashita, H., Buat, V., \& Inoue, A. K. 2003, A\&A, 410, 83

Hoflich, P., Khokhlov, A., \& Wheeler, J. C. 1995, ApJ, 444, 831

Howell, D. A. 2001, ApJ, 554, 193

Iben, I., \& Renzini, A. 1983, ARA\&A, 21, 271

Jarrett, T. H., Chester, T., Cutri, R., Schneider, S., \& Huchra, J. 2003, AJ, 125, 525

Kauffmann, G., Heckman, T. M., White, S. D. M., et al. 2003, MNRAS, 341, 33

Kennicutt, R. C., Jr. 1998, ARA\&A, 36, 189

Kroupa, P. 2001, MNRAS, 322, 231

Li, W., Filippenko, A. V., Treffers, R. R., et al. 2001, ApJ, 546, 743

Livio, M. 2001, in The greatest Explosions since the Big Bang: Supernovae and Gamma-Ray Bursts, ed. M. Livio, N. Panagia \& K. Sahu, Space Telescope Science Institute Symp. Ser. (Cambridge University Press), 13, 334

Livio, M., \& Riess, A. G. 2003, ApJ, 594, 93
Madau, P., Della Valle, M., \& Panagia, N. 1998, MNRAS, 297, 17

Mannucci, F., Maiolino, R., Cresci, G., et al. 2003, A\&A, 401, 519

Nomoto, K., Uenishi, T., Kobayashi, C., et al. 2003, in From Twilight to Highlight: the Physics of Supernovae, ed. W. Hillebrandt, \& B. Leibudgut, ESO/Springer Ser., ESO Astrophys. Symp., 115 [arXiv: astro-ph/0308138]

Nugent, P., Baron, E., Branch, D., Fisher, A., \& Hauschildt, P. 1997, ApJ, 485, 812

Oemler, A., \& Tinsley, B. 1979, AJ, 84, 985

Panagia, N. 2000, in Experimental Physics of Gravitational Waves, ed. M. Barone, G. Calamai, M. Mazzoni, R. Stanga, \& F. Vetrano (Singapore: World Scientific Publishing Co. Pte. Ltd.), 107

Prugniel, P., \& Héraudeau, P., 1998, A\&AS, 128, 299

Paturel, G., Fouque, P., Bottinelli, L., \& Gouguenheim, L. 1989, A\&AS, 80, 299

Phillips, M. M. 1993, ApJ, 413, L105

Pozzetti, L., Bruzual, A. G., \& Zamorani, G. 1996, MNRAS, 281, 953

Ruiz-Lapuente, P., Burkert, A., \& Canal, R. 1995, ApJ, 447, 69

Salpeter, E. E. 1955, ApJ, 121, 161

Saracco, P., Longhetti, M., Giallongo, E., et al. 2004, A\&A, 420, 125

Tammann, G. A. 1974, in Supernovae and Supernova remnants, ed. C. Batalli Cosmovici, 155

Tammann, G. A., Löffler, W., \& Schröder, A. 1994, ApJS, 92, 487

Turatto, M., Cappellaro, E., \& Benetti, S. 1994, AJ, 108, 202

Turatto, M., Benetti, S., Cappellaro, E., et al. 1996, MNRAS, 283, 1

van den Bergh, S. 1990, PASP, 102, 1318

van den Bergh, S., \& Tammann, G. A. 1991, ARA\&A, 29, 363

Wheeler, J. C., \& Harkness, R. P. 1993, Rep. Prog. Phys., 53, 1467

Woosley, S. E., \& Weaver, T. A. 1986, ARA\&A, 24, 205

Woosley, S. E., Heger, A., \& Weaver, T. A. 2002, Rev. Mod. Phys., 4, 1015

Yungelson, L. R., \& Livio, M. 2000, ApJ, 528, 108

Yungelson, L. R., \& Livio, M., Tutukov, A. V., \& Saffer, R. A. 1994, ApJ, 420, 336

Zwicky, F. 1942, ApJ, 96, 28 